\title{
Modelling population processes with random initial conditions
}

\author{
P.K. Pollett* $\quad$ A.H. Dooley ${ }^{\dagger} \quad$ J.V. Ross ${ }^{\ddagger}$
}

6th November 2009

\begin{abstract}
Population dynamics are almost inevitably associated with two predominant sources of variation: the first, demographic variability, a consequence of chance in progenitive and deleterious events; the second, initial state uncertainty, a consequence of partial observability and reporting delays and errors. Here we outline a general method for incorporating random initial conditions in population models where a deterministic model is sufficient to describe the dynamics of the population. Additionally, we show that for a large class of stochastic models the overall variation is the sum of variation due to random initial conditions and variation due to random dynamics, and thus we are able to quantify the variation not accounted for when random dynamics are ignored. Our results are illustrated with reference to both simulated and real data.
\end{abstract}

Keywords: Population processes; Epidemic models; Stochastic models.

\footnotetext{
*Prof. P.K. Pollett, Department of Mathematics, The University of Queensland, Qld 4072, Australia. ${ }^{\dagger}$ Prof. A.H. Dooley, School of Mathematics, University of New South Wales, Sydney 2052, Australia. ${ }^{\ddagger}$ Dr J.V. Ross, King’s College, University of Cambridge, Cambridge CB2 1ST, UK.
} 


\section{Introduction}

Often the initial state of population processes is not known with certainty. This can happen because of delays and inaccuracies in reporting, partial observability, and difficulties in assessing the actual population size. The initial state is often assumed to be known, or approximated and then treated as known, in which case mathematical models can give only partial information about dynamics and do not describe the full range of behaviour that may be exhibited. This may lead to inaccurate predictions and result in the implementation of non-optimal control actions.

Kegan and West [25] addressed this issue, in the context of the SI (susceptible-infectious) epidemic model, by investigating the effect of random initial conditions on the state of the deterministic SI model. Using the Beta distribution to model the initial proportion of infectives, they obtained explicit information about the distribution of the proportion of susceptibles at any time during the epidemic, as well as the distribution of the time until a given proportion of the population remains susceptible. We explain how their approach can be extended to allow initial state uncertainty to be incorporated in general population processes where a deterministic model is sufficient to describe the dynamics of the population, thus allowing one to model variability in dynamics, at any point in time, due solely to uncertainty in the initial state. For one-dimensional processes, such as those used for modelling sexually transmitted infections [2] and hospital-acquired infections [37], and a range of single-species population models, we demonstrate how this can be effected without the need to exhibit the trajectories explicitly. We illustrate our results with reference to several population models.

We also consider a wide class of stochastic models, called density-dependent processes, for which there are natural deterministic analogues, and summarise results that quantify variation not accounted for when demographic variability is ignored. Many models appearing in the ecology and epidemiology literature belong to this class. Our purpose here is simply to delineate and quantify variation due to initial state uncertainty and variation due to random dynamics, rather than to study the approximations in detail. The ability to delineate variability in this way has been shown to be of great importance in understanding both population and disease dynamics $[13,17,20,32]$. We illustrate these results for several population models, including 
a detailed study of simulated data from a model for disease spread in metapopulations, and real data on the prevalence of HIV antibodies in homosexual men [3, 25].

\section{Initial state uncertainty in deterministic models}

We outline how to account for initial state uncertainty in a general population modelling context. Let $n_{t}$ be the state of our process at time $t$, and assume that $n_{t}$ lies in some subset $S$ of $\mathbb{Z}^{D}$ (the $D$-dimensional integer lattice). This would typically be a vector of numbers of individuals of various types ( $D$ in total). Suppose also that there is a parameter $N$, which would usually be related to the size of the system (for example $N$ might be a population ceiling), such that $x_{t}=n_{t} / N$ can be interpreted as a vector of population densities, and that we have identified an appropriate deterministic model for $x_{t}$. We will suppose that $x_{t}=x_{t}\left(x_{0}\right)$ satisfies the ordinary differential equation

$$
x_{s}^{\prime}=F\left(x_{s}\right) \quad\left(x_{s} \in E, 0 \leq s \leq t\right),
$$

where the function $F: E \rightarrow \mathbb{R}^{D}$ is specified and $E$ is an appropriate subset of $\mathbb{R}^{D}(D$ dimensional Euclidean space). For example, if the state $x_{t}$ were a vector of proportions, such as the proportions of individuals of various types, then it would be natural to take $E=[0,1]^{D}$.

We will exploit the standard change of variable technique to assess the effect of assuming that the initial state is random. Let $X=\left(X_{1}, \ldots, X_{D}\right)$ be a $D$-dimensional random vector whose probability density function (pdf) $f_{X}$ is specified, and let $g: \mathbb{R}^{D} \rightarrow \mathbb{R}^{D}$ be an injective (one-to-one) map with continuous first partial derivatives. Denote by $\partial g(x)$ the Jacobian matrix $\left(\partial g_{i}(x) / \partial x_{j}\right)$. Then, the pdf of $Y=\left(Y_{1}, \ldots, Y_{D}\right)$, where $Y=g(X)$, is given by $f_{Y}(y)=|J(y)| f_{X}\left(g^{-1}(y)\right), y \in \mathbb{R}^{D}$, where $J(y)$ is the Jacobian of $g^{-1}(y)$ (the determinant of $\left.\partial g^{-1}(y)\right)$ and $|J(y)|$ is its absolute value (see for example [21, Section 4.7]). If the map is not injective, then it is usually possible to partition the domain into regions over which the map is injective.

Now think of the initial state as being a random variable $X_{0}$ with a specified pdf $f_{0}$. In determining the action of the map $g_{t}\left(x_{0}\right)=x_{t}\left(x_{0}\right)$ (for simplicity, assumed to be injective) on $f_{0}$, we obtain a pdf $f_{t}$ that summarises the effect of random initial conditions in our 
population: for any $t>0$,

$$
f_{t}(y)=\left|J_{t}(y)\right| f_{0}\left(g_{t}^{-1}(y)\right) \quad\left(y \in \mathcal{R}_{t}\right),
$$

where $J_{t}(y)$ is the Jacobian of $g_{t}^{-1}(y)$ and $\mathcal{R}_{t}=g_{t}(E)$ is the image of $E$ under $g_{t}$. We emphasise that $f_{t}$ is the pdf of the state of our process at time $t$, assuming deterministic dynamics with an initial pdf $f_{0}$.

Since our trajectory satisfies (1) with $F$ specified, we can often take this a step further. In the one-dimensional case $(D=1)$ we can exhibit $f_{t}$ explicitly. Let $L(u)$ be the primitive $L(u)=\int^{u} d w / F(w)$. Suppose $L$ is injective, so that $L^{-1}$ is well defined (it is sufficient that $F$ be everywhere positive or everywhere negative). Then, the solution to (1) can be written $x_{t}\left(x_{0}\right)=L^{-1}\left(t+L\left(x_{0}\right)\right)$. The Jacobian can also be evaluated. Since $g_{t}(x)=y$ if and only if $L(y)-t=L(x)$, we get $g_{t}^{-1}(y)=L^{-1}(L(y)-t)\left(=g_{-t}(y)\right)$, and so

$$
J_{t}(y)=\frac{d}{d y} L^{-1}(L(y)-t)=\left(L^{-1}\right)^{\prime}(L(y)-t) L^{\prime}(y)=\frac{L^{\prime}(y)}{L^{\prime}\left(L^{-1}(L(y)-t)\right)}=\frac{F\left(L^{-1}(L(y)-t)\right)}{F(y)},
$$

where here we have used the inverse function theorem, $\left(L^{-1}\right)^{\prime}(y)=1 /\left(L^{\prime}\left(L^{-1}(y)\right)\right)$, together with $L^{\prime}=1 / F$. Therefore, from (2),

$$
f_{t}(y)=\left|\frac{F\left(L^{-1}(L(y)-t)\right)}{F(y)}\right| f_{0}\left(L^{-1}(L(y)-t)\right) .
$$

We will illustrate these results in Section 5.

The corresponding expected value $m_{t}$ and covariance matrix $V_{t}$ of the state of the process at time $t$ can be evaluated either directly from (2) or from (3), or via the trajectory $x_{t}\left(x_{0}\right)$ :

$$
m_{t}=\mathbb{E} x_{t}\left(X_{0}\right)=\int_{E} x_{t}(u) f_{0}(u) d u
$$

and, taking $x_{t}(u)$ and $m_{t}$ to be row vectors,

$$
V_{t}=\operatorname{Cov}\left(x_{t}\left(X_{0}\right)\right)=\int_{E} x_{t}(u)^{\top} x_{t}(u) f_{0}(u) d u-m_{t}^{\top} m_{t},
$$

where ${ }^{\top}$ denotes transpose.

One might expect that in most instances $f_{t}$ would be a poor model for the state of the population at time $t$, because randomness in the dynamics of the process (demographic variability) is not taken into account. The effect of ignoring random dynamics would be particularly pronounced when $t$ becomes large, for it quickly becomes the only source of variation. Suppose 
that the trajectory $x_{t}\left(x_{0}\right)$ approaches an equilibrium point $x_{\mathrm{eq}}$ of (1) (that is, $F\left(x_{\mathrm{eq}}\right)=0$ and $x_{\mathrm{eq}}$ is stable). Then, it is clear from (4) and (5) that, under mild conditions (for example, $x_{t}(u)$ monotonic in $t, \mathbb{E} x_{t}\left(X_{0}\right)<\infty$ or $E$ finite), $m_{t} \rightarrow x_{\text {eq }}$ and $V_{t} \rightarrow 0$ as $t \rightarrow \infty$ (that is, the randomness induced by the initial distribution disappears). Thus, it would be useful to quantify demographic variation and to be able to delineate this and initial state uncertainty. This will be achieved for a wide class of stochastic population models, termed density-dependent, using properties of conditional expectation coupled with the idea of a diffusion approximation, whereby the process is approximated by a simpler one (a Gaussian diffusion) whose properties can be exhibited explicitly in terms of the parameters of the original model.

\section{Density-dependent population models}

Our population process $\left(n_{t}, t \geq 0\right)$ is now assumed to be a continuous-time Markov chain with state space $S \subseteq \mathbb{Z}^{D}$. We let $q(m, n)$ denote the rate at which the process moves from state $m$ to state $n$ for $n \neq m$ and set $q(m, m)=-q(m)$, where $q(m)=\sum_{n \neq m} q(m, n)(<\infty)$ is the total rate at which the process leaves state $m$.

We will suppose that population process is density dependent in the sense of Kurtz [26]: there is a parameter $N$ with the property

$$
q(n, n+l)=N f\left(\frac{n}{N}, l\right) \quad(n, n+l \in S),
$$

for suitable functions $f(x, l), x \in E$, where $E$ is a subset of $\mathbb{R}^{D}$. As before, $N$ will usually be related to the size of the system and $n / N$ will usually be interpreted as a population density (or vector of population densities). Condition (6) stipulates that $n_{t}$ changes at a rate that depends on $n_{t}$ only through (the density) $X_{t}=n_{t} / N$, a property shared by a wide variety of models that arise in areas as diverse as ecology [40, 42, 43, 45], epidemiology [6, 12, 48],

parasitology [38], chemical kinetics [4, 28, 35, 41], telecommunications [39, 46] and random graphs $[14,52]$. Notice that the density process $\left(X_{t}, t \geq 0\right)$, being itself a Markov chain, takes values in the set $E$ no matter what the value of $N$.

Before proceeding, we note that there is a larger class of models termed asymptotically density dependent [38], where more general dependence on $N$ is permitted, but which disappears in the limit as $N$ gets large. All of the results presented below carry over without change. 
Now, a formal argument based on the forward equations (the master equation) for state probabilities, shows that $(d / d t) \mathbb{E} X_{t}=\mathbb{E} F\left(X_{t}\right)$, where

$$
F(x)=\sum_{l \neq 0} l f(x, l) \quad(x \in E),
$$

suggesting that (6) entails a law of motion of the kind (1) for the mean path $m_{t}=\mathbb{E} X_{t}$. However, it is not generally true that $m_{t}^{\prime}=F\left(m_{t}\right)$. For example, the SI model has $F(x)=$ $-\beta x(1-x), x \in E$, where $\beta$ is the transmission rate (see Section 5 ), and therefore $m_{t}^{\prime}=$ $F\left(m_{t}\right)+\beta \operatorname{Var}\left(X_{t}\right)$. This was observed by Isham [23] and exploited in West and Thompson's study [51], where they argued that the variance is small when the initial number of susceptibles is large. In fact this is true in great generality, being a consequence of the basic limit theorems of Kurtz [26, 27], which we will now describe. These results allow us to identify the most appropriate approximating deterministic model and to quantify variation not accounted for when random dynamics are ignored. As $N$ will vary, we need to make the dependence on $N$ explicit in our notation; we write $X_{t}^{(N)}=n_{t} / N$.

Suppose that $F$, given by (7), is Lipschitz on $E$, that is, for some positive constant $K_{E}$, $|F(x)-F(y)|<K_{E}|x-y|, x, y \in E$ (true for example if $F$ has a bounded first derivative on $E$ ). This guarantees a unique solution $x_{t}=x_{t}\left(x_{0}\right)$ to (1) for any given initial value $x_{0}$.

Next we impose two technical conditions. They are: (i) $\sup _{x \in E} \sum_{l \neq 0}|l| f(x, l)<\infty$, and (ii) $\lim _{d \rightarrow \infty} \sup _{x \in E} \sum_{|l|>d}|l| f(x, l)=0, x \in E$ (notice that if $S$ is a finite set, which is typically the case for population processes, both will automatically be satisfied). Then (Theorem 3.1 Kurtz [26]), if $\lim _{N \rightarrow \infty} X_{0}^{(N)}=x_{0}$, the density process $\left(X_{t}^{(N)}, t \geq 0\right)$ converges uniformly in probability on $[0, t]$ to the deterministic trajectory starting at $x_{0}$, that is, for every $\epsilon>0$, $\operatorname{Pr}\left(\sup _{0 \leq s \leq t}\left|X_{s}^{(N)}-x_{s}\left(x_{0}\right)\right|>\epsilon\right) \rightarrow 0$ as $N \rightarrow \infty$. This functional law of large numbers establishes that the density process can be approximated by a limiting deterministic trajectory, provided that they start sufficiently close to one another. It is important to realize that for a given density-dependent population model the deterministic analogue is identified and convergence (on finite time intervals) to its trajectory is established.

However, for $N$ moderately large there will usually be sizeable variation about the deterministic path. Kurtz [27] provides results which allow us to quantify this variation precisely. Theorem 3.5 of [27] is a functional central limit law, which establishes, under extra mild con- 
ditions, that the fluctuations about the deterministic trajectory follow a Gaussian diffusion. Define a process $\left(Z_{t}^{(N)}, t \geq 0\right)$ by

$$
Z_{t}^{(N)}=\sqrt{N}\left(X_{t}^{(N)}-x_{t}\left(x_{0}\right)\right)
$$

In addition to the conditions stated earlier, suppose that (iii) $\lim _{d \rightarrow \infty} \sup _{x \in E} \sum_{|l|>d}|l|^{2} f(x, l)=$ 0 . Suppose also that $F$ has uniformly continuous first partial derivatives on $E$, and that the $D \times D$ matrix $G(x)$, defined by $G_{i j}(x)=\sum_{l \neq 0} l_{i} l_{j} f(x, l), x \in E$, is uniformly continuous on $E$. Then, if $\lim _{N \rightarrow \infty} \sqrt{N}\left(X_{0}^{(N)}-x_{0}\right)=z$, the process $\left(Z_{t}^{(N)}\right)$ converges weakly in $D[0, t]$ (the space of right continuous functions with left limits, defined on $[0, t]$ ) to a Gaussian diffusion $\left(Z_{s}, s \geq 0\right)$ with initial value $Z_{0}=z$ and with mean $\mathbb{E} Z_{s}=M_{s} z$, where $M_{s}=\exp \left(\int_{0}^{s} B_{u} d u\right)$ and $B_{u}=\partial F\left(x_{u}\left(x_{0}\right)\right)$, and covariance

$$
\Sigma_{s}\left(x_{0}\right)=\operatorname{Cov}\left(Z_{s}\right)=M_{s} \int_{0}^{s} M_{u}^{-1} G\left(x_{u}\left(x_{0}\right)\right)\left(M_{u}^{-1}\right)^{\top} d u M_{s}^{\top},
$$

being the unique solution to

$$
\frac{d}{d s} \Sigma_{s}\left(x_{0}\right)=B_{s} \Sigma_{s}\left(x_{0}\right)+\Sigma_{s}\left(x_{0}\right) B_{s}^{\top}+G\left(x_{s}\left(x_{0}\right)\right)
$$

with $\Sigma_{0}\left(x_{0}\right)=0$. The idea is to use the diffusion process, whose parameters can often be determined explicitly, to approximate quantities for the original process by way of (8) - hence the term diffusion approximation. In particular, for all $t>0, X_{t}^{(N)}$ has an approximate $(D$ dimensional) normal distribution with $\operatorname{Cov}\left(X_{t}^{(N)}\right) \simeq \Sigma_{t}\left(x_{0}\right) / N$, where $\Sigma_{t}\left(x_{0}\right)$ is given by (9). We would usually take $X_{0}^{(N)}=x_{0}$, giving $\mathbb{E} X_{t}^{(N)} \simeq x_{t}\left(x_{0}\right)$. The quality of these approximations improves as $N$ gets large.

An important special case of the above is when $x_{0}$ is chosen as an equilibrium point (usually a stable equilibrium) of the deterministic model (so $x_{t}\left(x_{0}\right)=x_{0}$ for all $t$ ). Then, the approximating diffusion is an Ornstein-Uhlenbeck process (OU process), and more precise results are available [47]. In particular, if $F\left(x_{\mathrm{eq}}\right)=0$ and $B=\partial F\left(x_{\mathrm{eq}}\right)$, our approximating normal distribution has $\mathbb{E} X_{t}^{(N)} \simeq x_{\text {eq }}$ and $\operatorname{Cov}\left(X_{t}^{(N)}\right) \simeq \Sigma_{t} / N$, where

$$
\Sigma_{t}=\int_{0}^{t} e^{B u} G\left(x_{\mathrm{eq}}\right) e^{B^{\top} u} d u=\Sigma_{\mathrm{eq}}-e^{B t} \Sigma_{\mathrm{eq}} e^{B^{\top} t}
$$

and $\Sigma_{\text {eq }}$ satisfies $B \Sigma_{\text {eq }}+\Sigma_{\text {eq }} B^{\top}+G\left(x_{\text {eq }}\right)=0$, being the stationary solution to (10). For example, in the one-dimensional case $(D=1)$, one obtains the very simple expression $\Sigma_{t}=$ 
$\left(G\left(x_{\text {eq }}\right) /(-2 B)\right)\left(1-e^{2 B t}\right)$. One would use (11) in place of (9) when $t$ is large and $N$ is not so large that random dynamics can be ignored. While important in general population modelling contexts, it is less important in epidemiological modelling, for there one is usually interested in assessing the progress of a disease at times well before equilibrium (endemicity) is reached.

We emphasise that $\Sigma_{t}\left(x_{0}\right) / N$ measures the variation at time $t$ not accounted for when random dynamics only are ignored. It can thus be used to assess whether a deterministic model will suffice or whether the full stochastic model is needed. For further details and extensions of these methods, see $\operatorname{Kurtz}[29,30]$, Barbour [5, 7, 8, 9] and, more recently, Darling and Norris [15].

We now have a straightforward means of quantifying demographic variation for density dependent models. Next we examine how to delineate demographic variation and initial state uncertainty.

\section{Initial state uncertainty and random dynamics}

We will use conditional expectation. Our functional law of large numbers permits us to approximate the expected state at any time $t$ by the deterministic trajectory $x_{t}\left(x_{0}\right)$ starting at a fixed state $x_{0}$ : for large $N, \mathbb{E}\left(X_{t} \mid X_{0}=x_{0}\right) \simeq x_{t}\left(x_{0}\right)$. If the initial state is now a random variable $X_{0}$, we have $\mathbb{E} X_{t}=\mathbb{E} \mathbb{E}\left(X_{t} \mid X_{0}\right) \simeq \mathbb{E} x_{t}\left(X_{0}\right)=m_{t}$ (refer to (4)). For the covariance of the state at time $t$ we use the identity $\operatorname{Cov}\left(X_{t}\right)=\operatorname{Cov}\left(\mathbb{E}\left(X_{t} \mid X_{0}\right)\right)+\mathbb{E} \operatorname{Cov}\left(X_{t} \mid X_{0}\right)$, which follows from basic properties of conditional expectation. It shows, in effect, that if the diffusion approximation is employed the overall variation is the sum of variation due to deterministic dynamics under random initial conditions and the variation due to random dynamics, for the first term is approximately $\operatorname{Cov}\left(x_{t}\left(X_{0}\right)\right)=V_{t}$ (refer to (5)), and, under the diffusion approximation, $\operatorname{Cov}\left(X_{t} \mid X_{0}=x_{0}\right) \simeq \Sigma_{t}\left(x_{0}\right) / N$, where $\Sigma_{t}\left(x_{0}\right)$ is given by (9). Therefore,

$$
\operatorname{Cov}\left(X_{t}\right) \simeq V_{t}+\frac{1}{N} \int_{E} \Sigma_{t}(u) f_{0}(u) d u
$$

The second term on the right-hand side measures the variation not accounted for when using a deterministic model with initial state uncertainty. It is asymptotically negligible in the limit as $N \rightarrow \infty$. Thus evaluation of this term when the initial state is not known with certainty 
helps us decide whether a deterministic model will suffice, or if the full stochastic model is needed.

\section{Investigation}

We first consider the SI (susceptible-infectious) model, used to study diseases for which there is no recovery following infection; examples of such are HIV/AIDS, Hepatitis C for the majority of carriers, and many plant diseases. The model is also often used to study the initial stages of emerging infectious disease growth in a single community (see for example [44]). We can assess the degree of uncertainty not accounted for when deterministic SI model is used in place of the stochastic model, and when there is uncertainty in the initial state of the disease process or demographic variability in the population at risk.

The SI model. Our state $n_{t}$ is the number of susceptibles at time $t$. We may take $S=$ $\{0,1, \ldots, N-1\}$, where $N$ is the total number of individuals, for we will assume that there is at least one infective. The process has non-zero transition rates $q(n, n-1)=(\beta / N) n(N-n)$, where $\beta$ is the transmission rate. The model is density dependent with $f(x,-1)=\beta x(1-x)$, $x \in E=[0,1]$, and so the approximating deterministic model for the proportion $X_{t}^{(N)}=n_{t} / N$ of susceptibles is $(1)$ with $F(x)=-\beta x(1-x)$. It has the unique solution

$$
x_{t}\left(x_{0}\right)=x_{0} /\left(x_{0}+\left(1-x_{0}\right) e^{\beta t}\right) \quad(t \geq 0),
$$

for any given initial proportion $x_{0}$. Since $S$ is finite and $F^{\prime}$ exists and is bounded on $E$, uniform convergence in probability (over finite time intervals) of the process $X_{t}^{(N)}$ to the deterministic trajectory $x_{t}$ is guaranteed provided $X_{0}^{(N)} \rightarrow x_{0}$.

The primitive $L(u)=\int^{u} d w / F(w)$ can be evaluated as $L(u)=(1 / \beta) \log (1-1 / u)$ and its inverse is $L^{-1}(u)=1 /\left(1-e^{\beta u}\right)$. Therefore, $\left.L^{-1}(L(y)-t)\right)=y e^{\beta t} /\left(1-y+y e^{\beta t}\right)$, and so, from (3),

$$
f_{t}(y)=\frac{e^{\beta t}}{\left(1-y+y e^{\beta t}\right)^{2}} f_{0}\left(\frac{y e^{\beta t}}{1-y+y e^{\beta t}}\right) \quad(y \in E),
$$

the image of $E$ under $x_{0} \mapsto x_{t}\left(x_{0}\right)$ being $E$ itself (for all $t$ ). Thus, if $N$ is sufficiently large that random dynamics can be ignored, the pdf $f_{t}$ can be used to model the proportion of susceptibles at time $t$ given an initial pdf $f_{0}$. 
A commonly used distribution that captures uncertainty in the initial proportion of susceptible (or infectious) individuals is the $\operatorname{Beta}(a, b)$ distribution, because it has support $[0,1]$ and because of its amenability in shape:

$$
f_{0}(y)=\frac{\Gamma(a+b)}{\Gamma(a) \Gamma(b)} y^{a-1}(1-y)^{b-1} \quad(0 \leq y \leq 1)
$$

If the Beta distribution is employed, (14) leads to Equation (3) of Kegan and West [25]:

$$
f_{t}(y)=\frac{\Gamma(a+b)}{\Gamma(a) \Gamma(b)} e^{a \beta t} \frac{y^{a-1}(1-y)^{b-1}}{\left(1-y+y e^{\beta t}\right)^{a+b}} \quad(0 \leq y \leq 1) .
$$

If random dynamics are significant, we may employ the diffusion approximation. The SI model is a case where we can solve (9) explicity. We have that $G(x)=\beta x(1-x)=-F(x)$, and this leads to

$$
\Sigma_{t}\left(x_{0}\right)=e^{\beta t} x_{0}\left(1-x_{0}\right) \frac{\left(1-x_{0}\right)^{2} e^{2 \beta t}-\left(1-2 x_{0}-2 \beta t x_{0}\left(1-x_{0}\right)\right) e^{\beta t}-x_{0}^{2}}{\left(x_{0}+\left(1-x_{0}\right) e^{\beta t}\right)^{4}} .
$$

For fixed $x_{0}, X_{t}^{(N)}$ has an approximate normal distribution with mean $\mathbb{E} X_{t}^{(N)} \simeq x_{t}\left(x_{0}\right)$ and variance $\operatorname{Var}\left(X_{t}^{(N)}\right) \simeq \Sigma_{t}\left(x_{0}\right) / N$, the quality of the approximation improving as $N$ gets large.

If $N$ is small then one is forced to use the full stochastic model. Since the SI model is a pure-birth process one can evaluate $p_{n}(t)=\operatorname{Pr}\left(n_{t}=n\right)$ explicitly. It is easy to show that

$$
p_{n}(t)=\sum_{k=n}^{n_{0}} \frac{k(N-k)}{n(N-n)} e^{-(\beta / N) k(N-k) t} \prod_{l=n, l \neq k}^{n_{0}} \frac{l(N-l)}{(l-k)(N-l-k)} \quad\left(n=1, \ldots, n_{0}\right),
$$

with $p_{n}(0)$ being the complementary probability (tweak Exercise 6.8.31 of Grimmett and Stirzaker [21]).

For the SI model with $\beta=0.3$ and with the initial proportion of susceptibles following a $\operatorname{Beta}(8,1.5)$ distribution, we evaluated the difference between exact variance (evaluated numerically) and the variance obtained from mapping the initial distribution using the trajectory (13). Figure 1 shows the variability ignored, at times $t \in[0,15]$, along with the corresponding proportion of the total variation. Notice that the variation not accounted for decreases with increasing population size. It is also apparent that the unexplained variation can be a sizeable proportion of the total variation in the dynamics and that the proportion of variation unexplained increased over time; as the effect of the initial distribution dissipates, the variation predicted by the stochastic model predominates. One can thus make an informed 
decision about the use of the deterministic or the stochastic model through demarcation and quantification of variability. In particular, they allow for this assessment to be made at any stage in the progress of the infection.

We now consider disease trajectories from the stochastic SI model with transmission rate parameter $\beta=0.5$, a $\operatorname{Beta}(20,1)$ initial distribution and $N=50$ (Figure 2a). Here we can think of a metapopulation consisting of $N=50$ patches, in which a disease is spreading between these patches. It can be seen that there is sizeable variability between individual trajectories (red dashed with crosses, nine realisations). Furthermore, we can see that the deterministic model incorporating an initial distribution (blue lines; circles - mean, dashed two standard deviations) does not account for all of the variability in trajectories but does account for the majority of it. The corresponding stochastic model standard deviation bounds (black dashed) provide an improved estimate of the potential disease progression seen in reality; the standard deviation bounds arising from accounting for stochasticity only, that is, with no random initial conditions, also fail to account for all of the variability witnessed (green dashed). It is possible that the disease trajectory may be outside the bounds given by the deterministic model, as seen in four of the nine realisations shown, suggesting that the stochastic model incorporating an initial distribution might be used for pandemic and emerging infectious disease preparedness in this particular metapopulation scenario. Finally, we note that as time progresses the need to account for stochasticity, in concert with random initial conditions, increases.

An example used previously to illustrate incorporating an initial distribution for the SI model is early growth (1978-1984) of HIV prevalence in San Francisco [3, 25]. In Figure 2b we plot the available data (black filled circles) along with the corresponding mean (blue circles) and two standard error bounds (stochastic only - green dashed, deterministic with random initial conditions - blue dashed, stochastic with random initial conditions - black dashed). A Beta(3.4998, 0.2168) distribution was used for the initial proportion of susceptible individuals. It can be seen that since the population estimated to be at risk is large $(N=100,000)$, the additional variation due to random dynamics is small compared with the variability due to initial state uncertainty, as the deterministic and stochastic bounds almost coincide; this is reinforced by the bounds for the stochastic model with no initial uncertainty. In this scenario 
it may thus be sufficient to adopt the deterministic model with initial uncertainty for prediction. However, note that while the mean disease trajectory fits the observed data extremely well, individual realisations from the fitted model (Figure 2b: red dashed with crosses, seven realisations) provide a poor representation of the shape of the observed data. This illustrates the importance of using a stochastic model for model calibration and verification, even if a deterministic model is sufficient to give bounds on disease behaviour (as is the case here).

It is not surprising that the SI model provides a poor representation of HIV prevalence, as 'network' properties for such diseases have been shown to be of importance [16]. Bailey (1988) modified the model by dividing the population into two classes: a fraction that were not at risk due to a 'safe' lifestyle, and the remainder at risk. Here we consider an alternate approach to explain the observed data. We modify the SI model so that the transmission parameter $\beta$ decreases through time, following the identification of HIV in 1981-a modification to incorporate an element of rational/precautionary behaviour as awareness and perceived risk increases. This modified model provides realisations that give an accurate representation of the observed trajectory (Figure 2c: black filled circles - data; red dashed with red crosses five realisations from initial data proportion $)(\beta \leftarrow 0.99988 \beta$ following each new infection after 1981). Obviously, as already mentioned, specific network properties are important, along with demography (age structure); however, we also believe that this rational behavioural aspect of the model is an important and interesting component of the disease dynamics $[11,19]$.

Next we consider the SIS (Susceptible-Infective-Susceptible) model, which was one of the earliest stochastic models for the spread of infections that do not confer any long lasting immunity, and where individuals become susceptible again after infection $[18,50]$. It has been applied, not only in epidemiology, but also in the propagation of rumours [10], in chemical reaction kinetics [34] and in metapopulation ecology [40].

The SIS model. Our state $n_{t}$ is now the number of infectives at time $t$ and $S=\{0,1, \ldots, N\}$, where $N$ is the total number of individuals. The non-zero transition rates are $q(n, n+1)=$ $(\beta / N) n(N-n)$ and $q(n, n-1)=\nu n$, where $\beta$ is the transmission rate and $\nu$ is the recovery rate. The model is density dependent with $f(x,+1)=\beta x(1-x)$ and $f(x,-1)=\nu x, x \in E=[0,1]$, and so the approximating deterministic model for the proportion $X_{t}^{(N)}=n_{t} / N$ of infectives is (1) with $F(x)=\beta x(1-\rho-x)$, where $\rho=\nu / \beta$, being the classical Verhulst model [49] for 
population growth (see also $[36,31])$. It has the unique solution

$$
x_{t}\left(x_{0}\right)=\frac{(1-\rho) x_{0}}{x_{0}+\left(1-\rho-x_{0}\right) e^{-\kappa t}} \quad(t \geq 0)
$$

where $\kappa=\beta(1-\rho)$, if $\rho \neq 1$ and $x_{t}\left(x_{0}\right)=x_{0} /\left(1+\beta x_{0} t\right)(t \geq 0)$ if $\rho=1$. Again uniform convergence in probability (over finite time intervals) of the process $X_{t}^{(N)}$ to the deterministic trajectory $x_{t}$ is guaranteed provided $X_{0}^{(N)} \rightarrow x_{0}$. The primitive $L$ and its inverse are given by

$$
L(u)=\frac{1}{\kappa} \log \left(\frac{u}{1-\rho-u}\right) \quad \text { and } \quad L^{-1}(u)=\frac{1-\rho}{1+e^{-\kappa u}} \quad \text { if } \rho \neq 1,
$$

and $L(u)=L^{-1}(u)=1 /(\beta u)$ if $\rho=1$, and this leads to

$$
f_{t}(y)=\frac{(1-\rho)^{2} e^{-\kappa t}}{\left(1-\rho-y+y e^{-\kappa t}\right)^{2}} f_{0}\left(\frac{(1-\rho) y e^{-\kappa t}}{1-\rho-y+y e^{-\kappa t}}\right) \quad\left(y \in \mathcal{R}_{t}\right) \quad \text { if } \rho \neq 1,
$$

and

$$
f_{t}(y)=\frac{1}{(1-\beta t y)^{2}} f_{0}\left(\frac{y}{1-\beta t y}\right) \quad\left(y \in \mathcal{R}_{t}\right) \quad \text { if } \rho=1,
$$

where $\mathcal{R}_{t}$ is the interval $\left[0, x_{t}(1)\right]$. Note that, although $\mathcal{R}_{t}$ decreases to the point set $\{0\}$ as $t \rightarrow \infty$ if $\rho \geq 1$ and to the interval $[0,1-\rho]$ if $\rho<1$, the argument of $f_{0}$ in (15) and in (16) always lies in $(0,1)$. We might then take $f_{0}$ to be the $\operatorname{Beta}(a, b)$ pdf to obtain a model for the proportion of susceptibles at time $t$, assuming random dynamics can be ignored:

$$
f_{t}(y)=\frac{\Gamma(a+b)}{\Gamma(a) \Gamma(b)}(1-\rho)^{a+1} e^{-a \kappa t} \frac{y^{a-1}\left(1-\rho-y+\rho y e^{-\kappa t}\right)^{b-1}}{\left(1-\rho-y+y e^{-\kappa t}\right)^{a+b}} \quad\left(y \in \mathcal{R}_{t}\right) \quad \text { if } \rho \neq 1,
$$

and

$$
f_{t}(y)=\frac{\Gamma(a+b)}{\Gamma(a) \Gamma(b)} \frac{y^{a-1}(1-y-\beta t y)^{b-1}}{(1-\beta t y)^{a+b}} \quad\left(y \in \mathcal{R}_{t}\right) \quad \text { if } \rho=1 .
$$

We can again solve (9) explicity. We have that $G(x)=\beta x(1-x)+\nu x=F(x)+2 \nu x$, and a lengthy calculation gives

$$
\begin{aligned}
\Sigma_{t}\left(x_{0}\right) & =\frac{x_{0}}{\left(x_{0}+\left(1-\rho-x_{0}\right) e^{-\kappa t}\right)^{4}}\left(\rho x_{0}^{3}\left(1-e^{-2 \kappa t}\right)+(1+5 \rho) x_{0}^{2}\left(1-\rho-x_{0}\right) e^{-\kappa t}\left(1-e^{-\kappa t}\right)\right. \\
& \left.+2(1+2 \rho) x_{0}\left(1-\rho-x_{0}\right)^{2} \kappa t e^{-2 \kappa t}+(1+\rho)\left(1-\rho-x_{0}\right)^{3} e^{-2 \kappa t}\left(1-e^{-\kappa t}\right)\right) \quad \text { if } \rho \neq 1,
\end{aligned}
$$

and

$$
\Sigma_{t}\left(x_{0}\right)=\frac{\left(3 \beta^{3} x_{0}^{3} t^{3}+2 \beta^{2} x_{0}^{2} t^{2}\left(6-x_{0}\right)+6 \beta x_{0} t\left(3-x_{0}\right)+6\left(2-x_{0}\right)\right) \beta x_{0} t}{6\left(1+\beta x_{0} t\right)^{4}} \quad \text { if } \rho=1 .
$$


If random dynamics are significant, we may approximate the distribution of $X_{t}^{(N)}$ using a normal distribution with mean $x_{t}\left(x_{0}\right)$ and variance $\Sigma_{t}\left(x_{0}\right) / N$.

If $\rho \geq 1$ the infection will die out quickly. However if $\rho<1$ the process will move quickly towards the stable point $x_{\mathrm{eq}}=1-\rho$, in which case if $t$ is large and $N$ is not so large that random dynamics can be ignored we may employ the OU approximation: $X_{t}^{(N)}$ has an approximate normal distribution with mean $1-\rho$ and variance $\Sigma_{t} / N$, where $\Sigma_{t}=\rho\left(1-e^{-2 \kappa t}\right)$ (which settles quickly to $\Sigma_{\text {eq }}=\rho$ ).

The SIS model has been used in the study of metapopulations [40] to model the number of occupied habitat patches in a population network, where the transmission and recovery events correspond to colonization of unoccupied patches through dispersal of individuals from occupied patches and local extinction of occupied patches. Within this context, we examine next an elaboration of the model which accounts for an external source of colonization [1, 43].

A mainland-island metapopulation model. Now $n_{t}$ represents the number of occupied patches in a network consisting of $N$ habitat patches (imagine a network of islands). Each occupied patch becomes empty at rate $e$ (the per-patch local extinction rate), colonization of empty patches occurs at rate $c / N$ for each suitable pair consisting of an empty patch and a non-empty patch ( $c$ is the colonization rate), and immigration from an external source (the "mainland") occurs at rate $\alpha$ (the per-patch immigration rate). The non-zero transition rates are $q(n, n+1)=\alpha(N-n)+(c / N) n(N-n)$ and $q(n, n-1)=e n$. Again we have density dependence, now with $f(x,+1)=(\alpha+c x)(1-x)$ and $f(x,-1)=e x, x \in E=[0,1]$, and so the approximating deterministic model for the proportion $X_{t}^{(N)}=n_{t} / N$ of occupied patches is (1) with $F(x)=\alpha+(c-e-\alpha) x-c x^{2}$, a model identified by Hanski [22]. It has the unique solution

$$
x_{t}\left(x_{0}\right)=\phi+\theta \frac{x_{0}-\phi+\theta \tanh (\theta c t)}{\theta+\left(x_{0}-\phi\right) \tanh (\theta c t)} \quad(t \geq 0),
$$

where $\phi=(c-e-\alpha) /(2 c)$ and $\theta=\sqrt{\phi^{2}+\alpha / c}$ (see [43]). Furthermore,

$$
L(u)=\frac{1}{\theta c} \tanh ^{-1}\left(\frac{u-\phi}{\theta}\right) \quad \text { and } \quad L^{-1}(u)=\phi+\theta \tanh (\theta c u)
$$

leading to

$$
f_{t}(y)=\frac{\theta^{2} \operatorname{sech}^{2}(\theta c t)}{(\theta-(y-\phi) \tanh (\theta c t))^{2}} f_{0}\left(\phi+\theta \frac{y-\phi-\theta \tanh (\theta c t)}{\theta-(y-\phi) \tanh (\theta c t)}\right) \quad\left(y \in \mathcal{R}_{t}\right),
$$


where $\mathcal{R}_{t}$ is the interval $\left[x_{t}(0), x_{t}(1)\right]$. As before, we might then take $f_{0}$ to be the $\operatorname{Beta}(a, b)$ pdf to obtain a model for the proportion of occupied patches at time $t$, assuming random dynamics can be ignored.

If random dynamics are significant, we can again use a diffusion approximation, but, while it is feasible to evaluate $\Sigma_{t}\left(x_{0}\right)$ explicitly, the expression is too complicated to be of any practical use. However, for the present model the process moves quickly towards a region containing the stable point $x_{\mathrm{eq}}=\theta+\phi$ (being the unique equilibrium point in $E$ ), in which case if $t$ is large and $N$ is not so large that random dynamics can be ignored we may employ the OU approximation: $X_{t}^{(N)}$ has an approximate normal distribution with mean $\theta+\phi$ and variance $\Sigma_{t} / N$, where

$$
\Sigma_{t}=\frac{e(\theta+\phi)}{2 \theta c}\left(1-e^{-2 \theta c t}\right)
$$

which settles quickly to $\Sigma_{\text {eq }}=e(\theta+\phi) /(2 \theta c)$.

A further elaboration of the basic metapopulation model accounts for dynamics in habitat by allowing the number of patches suitable for occupancy to vary stochastically [42, 43]. Here we examine the case $c=0$ (not considered in detail by Ross), where colonization from within the network is insignificant compared with colonization from the mainland. This model is not only of interest in its own right, but also serves to illustrate how our results can be applied to a two-dimensional $(D=2)$ model.

A mainland-island metapopulation model incorporating habitat dynamics. The state at time $t$ is $\left(m_{t}, n_{t}\right)$, where $m_{t}$ is the number of patches suitable for occupancy and $n_{t}$ is the number occupied. The network contains a total of $N$ patches, and so the state space is $S=\{(m, n): 0 \leq n \leq m \leq N\}$. The non-zero transition rates are

$$
\begin{gathered}
q((m, n) ;(m+1, n))=r(N-m), \quad q((m, n) ;(m-1, n))=d(m-n), \\
q((m, n) ;(m-1, n-1))=d n, \quad q((m, n) ;(m, n+1))=\alpha(m-n) \\
\text { and } q((m, n) ;(m, n-1))=e n,
\end{gathered}
$$

where the two new parameters, $d$ and $r$, represent the (per-patch) rate at which patches are disturbed (and thus rendered unsuitable for occupancy) and the (per-patch) rate at which patches recover. Notice that if an occupied patch is disturbed it becomes unoccupied. For 
simplicity, we will assume that $r \neq \alpha+e$. The model is density dependent with

$$
\begin{gathered}
f((u, v) ;(+1,0))=r(1-u), \quad f((u, v) ;(-1,0))=d(u-v), \\
f((u, v) ;(-1,-1))=d v, \quad f((u, v) ;(0,+1))=\alpha(u-v) \\
\text { and } f((u, v) ;(0,-1))=e v,
\end{gathered}
$$

taking $E=\{(u, v): 0 \leq v \leq u \leq 1\}$, and so the approximating deterministic model for $X_{t}^{(N)}=\left(U_{t}^{(N)}, V_{t}^{(N)}\right)^{\top}$, where $U_{t}^{(N)}=m_{t} / N$ and $V_{t}^{(N)}=n_{t} / N$ are the proportion of suitable and occupied patches, is (1) with $x=(u, v)^{\top}$ and

$$
F(x)=F(u, v)=\left(\begin{array}{c}
r-(r+d) u \\
\alpha u-\kappa v
\end{array}\right)=\left(\begin{array}{l}
r \\
0
\end{array}\right)-A x, \quad \text { where } \quad A=\left(\begin{array}{cc}
r+d & 0 \\
-\alpha & \kappa
\end{array}\right),
$$

and $\kappa=\alpha+e+d$. Its solution $x_{t}=\left(u_{t}, v_{t}\right)^{\top}$ has components

$$
u_{t}\left(u_{0}, v_{0}\right)=u_{\mathrm{eq}}+\left(u_{0}-u_{\mathrm{eq}}\right) e^{-(r+d) t}
$$

and

$$
v_{t}\left(u_{0}, v_{0}\right)=v_{\text {eq }}+\left(v_{0}-v_{\text {eq }}\right) e^{-\kappa t}+\delta\left(u_{0}\right)\left(e^{-\kappa t}-e^{-(r+d) t}\right),
$$

where $u_{\mathrm{eq}}=r /(r+d)$ and $v_{\mathrm{eq}}=\alpha u_{\mathrm{eq}} / \kappa$ are the limiting $(t \rightarrow \infty)$ values, and $\delta(y)=$ $\alpha\left(y-u_{\mathrm{eq}}\right) /(r-e-\alpha)$. The trajectories are curves in $E$ satisfiying

$$
v_{t}=v_{\mathrm{eq}}+\left(v_{0}-v_{\mathrm{eq}}\right)\left(\frac{u_{t}-u_{\mathrm{eq}}}{u_{0}-u_{\mathrm{eq}}}\right)^{\frac{\kappa}{r+d}}+\frac{\alpha\left(u_{0}-u_{\mathrm{eq}}\right)}{r-e-\alpha}\left(\left(\frac{u_{t}-u_{\mathrm{eq}}}{u_{0}-u_{\mathrm{eq}}}\right)^{\frac{\kappa}{r+d}}-\frac{u_{t}-u_{\mathrm{eq}}}{u_{0}-u_{\mathrm{eq}}}\right) .
$$

We will now determine the action of the map $g_{t}\left(u_{0}, v_{0}\right)=x_{t}\left(u_{0}, v_{0}\right)$ on $f_{0}$, the joint pdf of the initial proportions $\left(U_{0}, V_{0}\right)$ of suitable and occupied patches, to obtain a pdf $f_{t}$ that summarises the effect of random initial conditions in our metapopulation. A simple calculation shows that the map is injective with

$$
g_{t}^{-1}(y)=g_{-t}(y)=\left(\begin{array}{c}
u_{\mathrm{eq}}+\left(y_{1}-u_{\mathrm{eq}}\right) e^{(r+d) t} \\
v_{\mathrm{eq}}+\left(y_{2}-v_{\mathrm{eq}}\right) e^{\kappa t}+\delta\left(y_{1}\right)\left(e^{\kappa t}-e^{(r+d) t}\right)
\end{array}\right) \quad\left(y=\left(y_{1}, y_{2}\right)^{\top} \in \mathcal{R}_{t}\right),
$$

where $\mathcal{R}_{t}=g_{t}(E)$, the image of $E$ under $g_{t}$, is the triangle with vertices $g_{t}(0,0), g_{t}(1,0)$ and $g_{t}(1,1)$. (Note that $\mathcal{R}_{t}$ decreases to $\left\{\left(u_{\text {eq }}, v_{\text {eq }}\right)\right\}$ as $t \rightarrow \infty$.) Clearly

$$
\partial g_{t}^{-1}(y)=\left(\begin{array}{cc}
e^{(r+d) t} & 0 \\
\frac{\alpha}{r-e-\alpha}\left(e^{\kappa t}-e^{(r+d) t}\right) & e^{\kappa t}
\end{array}\right)
$$


and so $J_{t}(y)=\left|\partial g_{t}^{-1}(y)\right|=e^{(r+d+\kappa) t}(>0)$. Therefore, from (2),

$$
f_{t}(y)=f_{t}\left(y_{1}, y_{2}\right)=e^{(r+d+\kappa) t} f_{0}\left(\begin{array}{c}
u_{\mathrm{eq}}+\left(y_{1}-u_{\mathrm{eq}}\right) e^{(r+d) t} \\
v_{\mathrm{eq}}+\left(y_{2}-v_{\mathrm{eq}}\right) e^{\kappa t}+\delta\left(y_{1}\right)\left(e^{\kappa t}-e^{(r+d) t}\right)
\end{array}\right) \quad\left(y \in \mathcal{R}_{t}\right) .
$$

For example, we might suppose that the initial proportion $V_{0}$ of suitable patches follows a $\operatorname{Beta}(a, b)$ law on $[0,1]$ and the initial proportion $U_{0}$ of occupied patches follows a $\operatorname{Beta}(p, q)$ law on $\left[0, V_{0}\right]$ :

$$
f_{0}(u, v)=\frac{\Gamma(a+b)}{\Gamma(a) \Gamma(b)} \frac{\Gamma(p+q)}{\Gamma(p) \Gamma(q)} v^{a-p-q}(1-v)^{b-1} u^{p-1}(v-u)^{q-1} \quad(0 \leq u \leq v \leq 1) .
$$

To illustrate this, consider the simplest case where $V_{0}$ is uniformly distributed on $[0,1]$ and $U_{0}$ is uniformly distributed on $\left[0, V_{0}\right]$, so that $f_{0}(u, v)=1 / v(0 \leq u \leq v \leq 1)$ :

$$
f_{t}\left(y_{1}, y_{2}\right)=\frac{e^{(r+d+\kappa) t}}{v_{\text {eq }}+\left(y_{2}-v_{\text {eq }}\right) e^{\kappa t}+\delta\left(y_{1}\right)\left(e^{\kappa t}-e^{(r+d) t}\right)} \quad\left(\left(y_{1}, y_{2}\right) \in \mathcal{R}_{t}\right) .
$$

To obtain the diffusion approximation observe that

$$
G(x)=G(u, v)=\left(\begin{array}{cc}
r+(d-r) u & d v \\
d v & \alpha u+(d+e-\alpha) v
\end{array}\right) .
$$

Because $\partial F(u, v)=-A$ is the same for all $(u, v)$, the evaluation of $\Sigma_{t}\left(x_{0}\right)$ is simpler:

$$
\Sigma_{t}\left(x_{0}\right)=\int_{0}^{t} e^{-A(t-u)} G\left(x_{u}\left(x_{0}\right)\right) e^{-A^{\top}(t-u)} d u .
$$

Furthermore,

$$
e^{-A t}=\left(\begin{array}{cc}
e^{-(r+d) t} & 0 \\
\frac{\alpha}{\alpha+e-r}\left(e^{-(r+d) t}-e^{-\kappa t}\right) & e^{-\kappa t}
\end{array}\right),
$$

and so $\Sigma_{t}\left(x_{0}\right)$ can in principle be evaluated explicitly. However, since the process moves quickly towards $x_{\text {eq }}=\left(u_{\text {eq }}, v_{\text {eq }}\right)^{\top}$, the OU approximation will usually suffice. The unique solution to $B \Sigma_{\text {eq }}+\Sigma_{\text {eq }} B^{\top}+G\left(x_{\text {eq }}\right)=0$, where $B:=\partial F\left(u_{\text {eq }}, v_{\text {eq }}\right)=-A$, is

$$
\Sigma_{\mathrm{eq}}=\left(\begin{array}{cc}
u_{\mathrm{eq}}\left(1-u_{\mathrm{eq}}\right) & v_{\mathrm{eq}}\left(1-u_{\mathrm{eq}}\right) \\
v_{\mathrm{eq}}\left(1-u_{\mathrm{eq}}\right) & v_{\mathrm{eq}}\left(1-u_{\mathrm{eq}}\right)\left(1+\frac{r(e+d)}{d \kappa}\right)
\end{array}\right) .
$$

Thus, if $t$ is large and $N$ is not so large that random dynamics can be ignored, $\left(U_{t}^{(N)}, V_{t}^{(N)}\right)$ will have an approximate bivariate normal distribution with mean $\left(u_{\mathrm{eq}}, v_{\mathrm{eq}}\right)$ and covariance matrix $\Sigma_{\text {eq }} / N$. 


\section{Concluding remarks}

We have seen that if the population size $N$ is large, the effect of demographic stochasticity becomes negligible, in which case a deterministic model will suffice and we may incorporate random initial conditions by way of (2) (or (3) in the one-dimensional case). If $N$ is not so large that random dynamics can be ignored, a stochastic model should be used. For density dependent models we may exploit diffusion approximation techniques to estimate characteristics of the stochastic model. If $N$ is small we are forced to evaluate the state probabilities, either analytically or numerically, using standard techniques, incorporating random initial conditions as appropriate. Whatever our approach, the effect of uncertainty in initial population size becomes insignificant in the long term $(t \rightarrow \infty)$. The following table contains a summary. 


\begin{tabular}{lll}
\hline \multicolumn{1}{c}{$N$} & $t$ & Model and approach \\
\hline large & small & Deterministic model with random initial conditions \\
large & large & Deterministic model: identify a stable equilibrium \\
moderate & small & Stochastic model: full diffusion approximation with ran- \\
& & dom initial conditions \\
moderate & large & Stochastic model: OU approximation \\
small & small & Stochastic model: solve forward equations \\
small & large & Stochastic model: evaluate the stationary distribution \\
& & or the quasi-stationary distribution \\
\hline
\end{tabular}

For density dependent models, we have shown that the overall variation is the sum of the variation due to random initial conditions and the variation due to random dynamics, and thus we have quantified the variation not accounted for when random dynamics are ignored (formula $(12))$.

Acknowledgements. We thank both referees for their careful reading of the manuscript, and for valuable comments that led to a significant improvement in the presentation. The work of Anthony Dooley and Phil Pollett is supported by the Australian Research Council Centre of Excellence for Mathematics and Statistics of Complex Systems. Joshua Ross acknowledges the support of King's College Cambridge (Zukerman Research Fellowship).

\section{References}

[1] D. Alonso, and A. McKane. Extinction dynamics in mainland-island metapopulations: An N-patch stochastic model. Bull. Math. Biol. 64, 913-958, 2002.

[2] H. Andersson and T. Britton. Stochastic Epidemic Models and Their Statistical Analysis, Springer Lectures Notes in Statistics, Vol. 151, Springer, Berlin, 2000.

[3] N.T.J. Bailey. Simplified modelling of the population dynamics of HIV/AIDS. J. Roy. Statist. Soc. Ser. A 151, 31-34, 1988. 
[4] K. Ball, T.G. Kurtz, L. Popovic and G. Rempala. Asymptotic analysis of multiscale approximations to reaction networks. Ann. Appl. Probab. 16, 1925-1961, 2006.

[5] A.D. Barbour. On a functional central limit theorem for Markov population processes. Adv. Appl. Probab. 6, 21-39, 1974.

[6] A.D. Barbour. The duration of the closed stochastic epidemic. Biometrika 62, 477-482, 1975 .

[7] A.D. Barbour. Quasi-stationary distributions in Markov population processes. Adv. Appl. Probab. 8, 296-314, 1976.

[8] A.D. Barbour. Density dependent Markov population processes. In (Eds. W. Jager, H. Rost and P. Tautu) Biological growth and spread: mathematical theories and applications (Proc. Conf., Heidelberg, 1979), Lecture Notes in Biomathematics, Vol. 38, Springer, Berlin, pp. 36-49, 1980.

[9] A.D. Barbour. Equilibrium distributions for Markov population processes. Adv. Appl. Probab. 12, 591-614, 1980.

[10] D.J. Bartholomew. Continuous time diffusion models with random duration of interest. J. Math. Sociol. 4, 187-199, 1976.

[11] M.C.J. Bootsma and N.M. Ferguson. The effect of public health measures on the 1918 influenza pandemic in U.S. cities. Proc. Nat. Academy Sci. 104, 7588-7593, 2007.

[12] D. Clancy, P.D. O'Neill and P.K. Pollett. Approximations for the long-term behaviour of an open-population epidemic model. Methodology Comput. Appl. Probab. 3, 75-95, 2001.

[13] T. Coulson, P. Rohani and M. Pascual. Skeletons, noise and population growth: the end of an old debate? Trends Ecol. Evol. 19, 359-364, 2004.

[14] R.W.R. Darling and J.R. Norris. Structure of large random hypergraphs. Ann. Appl. Probab. 15, 125-152, 2005.

[15] R.W.R. Darling and J.R. Norris. Differential equation approximations for Markov chains. Probab. Surv. 5, 37-79, 2008. 
[16] K.T.D. Eames and M.J. Keeling. Modeling dynamic and network heterogeneities in the spread of sexually transmitted diseases. Proc. Nat. Academy Sci. 99, 13330-13335, 2002.

[17] B.D. Elderd, V.M. Dukic and G. Dwyer. Uncertainty in predictions of disease spread and public health responses to bioterrorism and emerging diseases. Proc. Nat. Academy Sci. 103, 15693-15697, 2006.

[18] W. Feller. Die grundlagen der volterraschen theorie des kampfes ums dasein in wahrscheinlichkeitsteoretischer behandlung. Acta Biotheor. 5, 11-40, 1939.

[19] N. Ferguson. Capturing human behaviour. Nature 446, 733, 2007.

[20] B.T. Grenfell, K. Wilson, B.F. Finkenstådt, T.N. Coulson, S. Murray, S.D. Albon, J.M. Pemberton, T.H. Clutton-Brock and M.J. Crawley. Noise and determinism in synchronized sheep dynamics. Nature 394, 674-67, 1998.

[21] G.R. Grimmett and D.R. Stirzaker. Probability and Random Processes. Oxford University Press, New York, 3rd edition, 2001.

[22] I.A. Hanski Metapopulation Ecology. Oxford University Press, Oxford, 1999.

[23] V. Isham. Mathematical modelling of the transmission dynamics of HIV infection and AIDS: a review J. Roy. Statist. Soc. Ser. A 151,5-30, 44-49, 1988 (with discussion).

[24] M.J. Keeling and J.V. Ross. On methods for studying stochastic disease dynamics. J. Roy. Soc. Interface 5, 171-181, 2008.

[25] B. Kegan and R.W. West. Modeling the simple epidemic with deterministic differential equations and random initial conditions. Math. Biosci. 194, 217-231, 2005.

[26] T.G. Kurtz. Solutions of ordinary differential equations as limits of pure jump Markov processes. J. Appl. Probab. 7, 49-58, 1970.

[27] T.G. Kurtz. Limit theorems for sequences of jump Markov processes approximating ordinary differential processes. J. Appl. Probab. 8, 344-356, 1971. 
[28] T.G. Kurtz. The relationship between stochastic and deterministic models in chemical reactions. J. Chem. Phys. 57, 2976-2978, 1972.

[29] T.G. Kurtz. Limit theorems and diffusion approximations for density dependent Markov chains. Math. Prog. Study 5, 67-78, 1976.

[30] T.G. Kurtz. Strong approximation theorems for density dependent Markov chains. Stochastic Process. Appl. 6, 223-240, 1978.

[31] R. Levins. Some demographic and genetic consequences of environmental heterogeneity for biological control. Bull. Entom. Soc. Amer. 15, 237-240, 1969.

[32] A.J. McKane and T.J. Newman. Predator-prey cycles from resonant amplification of demographic stochasticity. Phys. Rev. Lett. 94, 218102, 2005.

[33] J.R. Norris. Markov Chains. Cambridge University Press, 1997.

[34] I. Oppenheim, K.E. Shuler and G.H. Weiss. Stochastic theory of nonlinear rate processes with multiple stationary states. Physica 88A, 191-214, 1977.

[35] R.W. Parsons and P.K. Pollett. Quasistationary distributions for some autocatalytic reactions. J. Statist. Phys. 46, 249-254, 1987.

[36] R. Pearl and L. Reed. On the rate of growth of population of the United States since 1790 and its mathematical representation, Proc. Nat. Academy Sci. 6, 275-288, 1920.

[37] I. Pelupessy, M.J.M. Bonten and O. Diekmann. How to assess the relative importance of different colonization routes of pathogens within hospital settings. Proc. Nat. Academy Sci. 99, 5601-5605, 2002.

[38] P.K. Pollett. On a model for interference between searching insect parasites. J. Austral. Math. Soc. 31, 133-150, 1990.

[39] P.K. Pollett. Diffusion approximations for a circuit switching network with random alternative routing. Austral. Telecom. Res. 25, 45-52, 1991. 
[40] P.K. Pollett Diffusion approximations for ecological models. In (Ed. F. Ghassemi) Proceedings of the International Congress on Modelling and Simulation, Vol.2, Modelling and Simulation Society of Australia and New Zealand, Canberra, Australia, pp. 843-848, 2001.

[41] P.K. Pollett and A. Vassallo. Diffusion approximations for some simple chemical reaction schemes. Adv. Appl. Probab. 24, 875-89, 1992.

[42] J.V. Ross. A stochastic metapopulation model accounting for habitat dynamics. J. Math. Biol. 52, 788-806, 2006.

[43] J.V. Ross. Stochastic models for mainland-island metapopulations in static and dynamic landscapes. Bull. Math. Biol. 68, 417-449, 2006.

[44] J.V. Ross, D.E. Pagendam and P.K. Pollett. On parameter estimation in population models II: multi-dimensional processes and transient dynamics. Theor. Pop. Biol. 75, $123-132,2009$.

[45] J.V. Ross, T. Taimre and P.K. Pollett. On parameter estimation in population models. Theor. Pop. Biol. 70, 498-510, 2006.

[46] J.V. Ross, T. Taimre and P.K. Pollett. Estimation for queues from queue length data. Queueing Systems 55, 131-138, 2007.

[47] S. Schach. Weak convergence results for a class of multivariate Markov processes. Ann. Math. Statist. 42, 451-465, 1971.

[48] A. Sani, D.P. Kroese and P.K. Pollett. Stochastic models for the spread of HIV in a mobile heterosexual population. Math. Biosci. 208, 98-124, 2007.

[49] P.F. Verhulst. Notice sur la loi que la population suit dans son accroissement. Corr. Math. et Phys. X, 113-121, 1838.

[50] G.H. Weiss and M. Dishon. On the asymptotic behaviour of the stochastic and deterministic models of an epidemic. Math. Biosci. 11, 261-265, 1971. 
[51] R.W. West and J.R. Thompson. Models for the simple epidemic. Math. Biosci. 141, 29-39, 1997.

[52] N.C. Wormald. Differential equations for random processes and random graphs. Ann. Appl. Probab. 5, 1217-1235, 1995. 


\section{Legends}

Figure 1. The unexplained variation (a) and the corresponding proportion of total variation not accounted for (b) at times $t \in[0,15]$ for different population sizes $N \in\{10,20,40,80\}$ in the SI model with transmission rate $\beta=0.3$ and a $\operatorname{Beta}(8,1.5)$ distribution for the initial state.

Figure 2. Metapopulation disease spread and HIV prevalence in San Francisco: (a) Nine realisations (red dashed, cross) of the SI model with transmission rate parameter $\beta=0.5$, a Beta $(20,1)$ distribution for the initial proportion of susceptible patches and $N=50$ patches, along with the mean (blue circles) and two standard error bounds (green dashed - stochastic only; blue dashed - deterministic with random initial conditions; black dashed - stochastic with random initial conditions). (b) Estimates of HIV prevalence from a representative sample of homosexual men in San Francisco 1978-1984 (black, filled circles), seven realisations (red dashed, cross) of the previously fitted SI model with transmission parameter $\beta=1.18$ and a $\operatorname{Beta}(3.4998,0.2168)$ distribution for the initial proportion of susceptibles and population size $N=100,000([3,25])$, along with the mean (blue circles) and two standard error bounds (green dashed - stochastic only; blue dashed - deterministic with random initial conditions; black dashed - stochastic with random initial conditions). (c) Five realisations (red dashed, cross) of a modification to the SI model used in (b) to incorporate rational/precautionary behaviour of individuals following the identification of HIV in $1981 ; \beta \leftarrow 0.99988 \beta$ following each new infection from 1982 onwards, $\beta=1$ initially, and all realisations commence from the estimated proportion of susceptible individuals in 1978. The data is once again plotted (black, filled circles). 
Figure 1 (a)

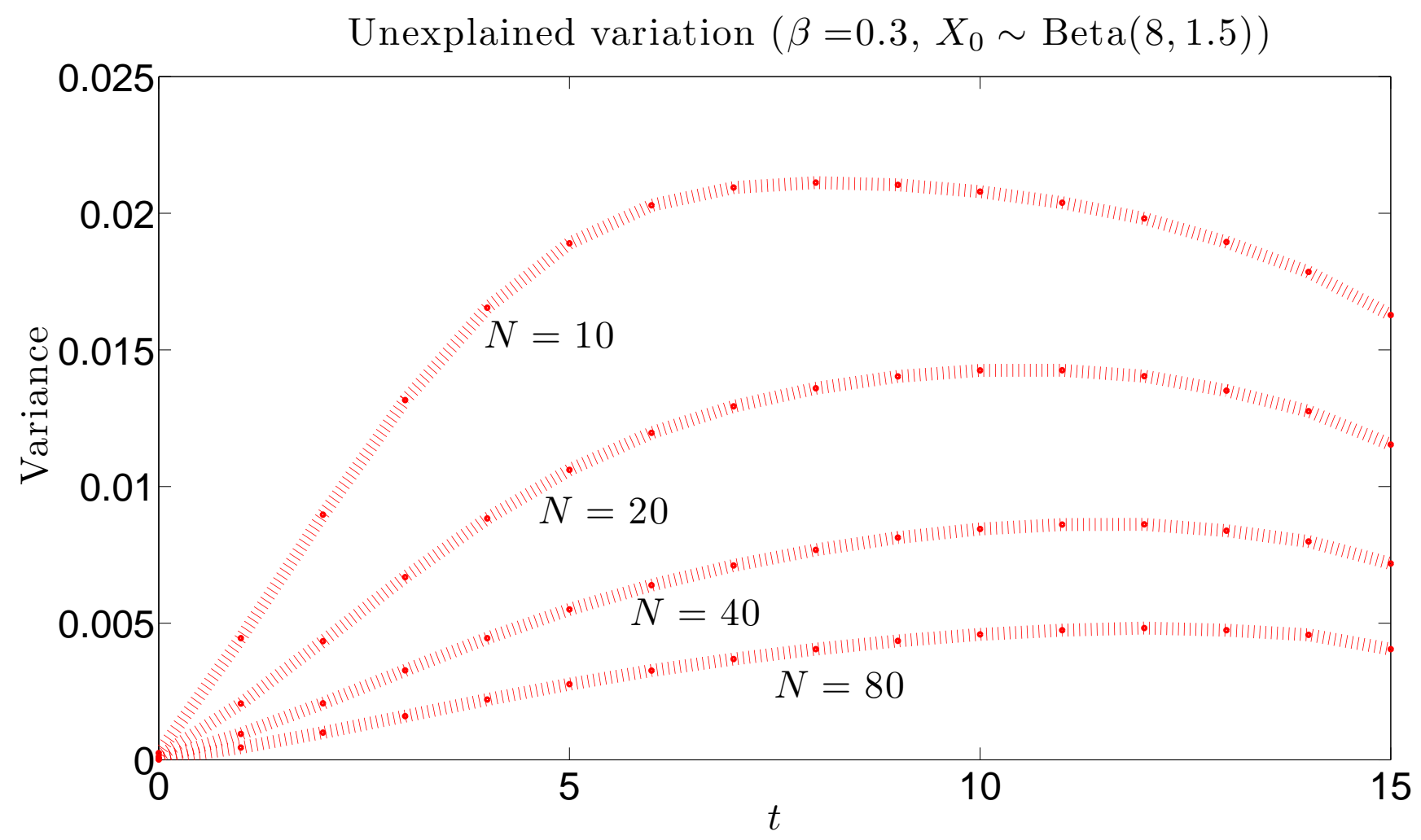

Figure 1 (b)

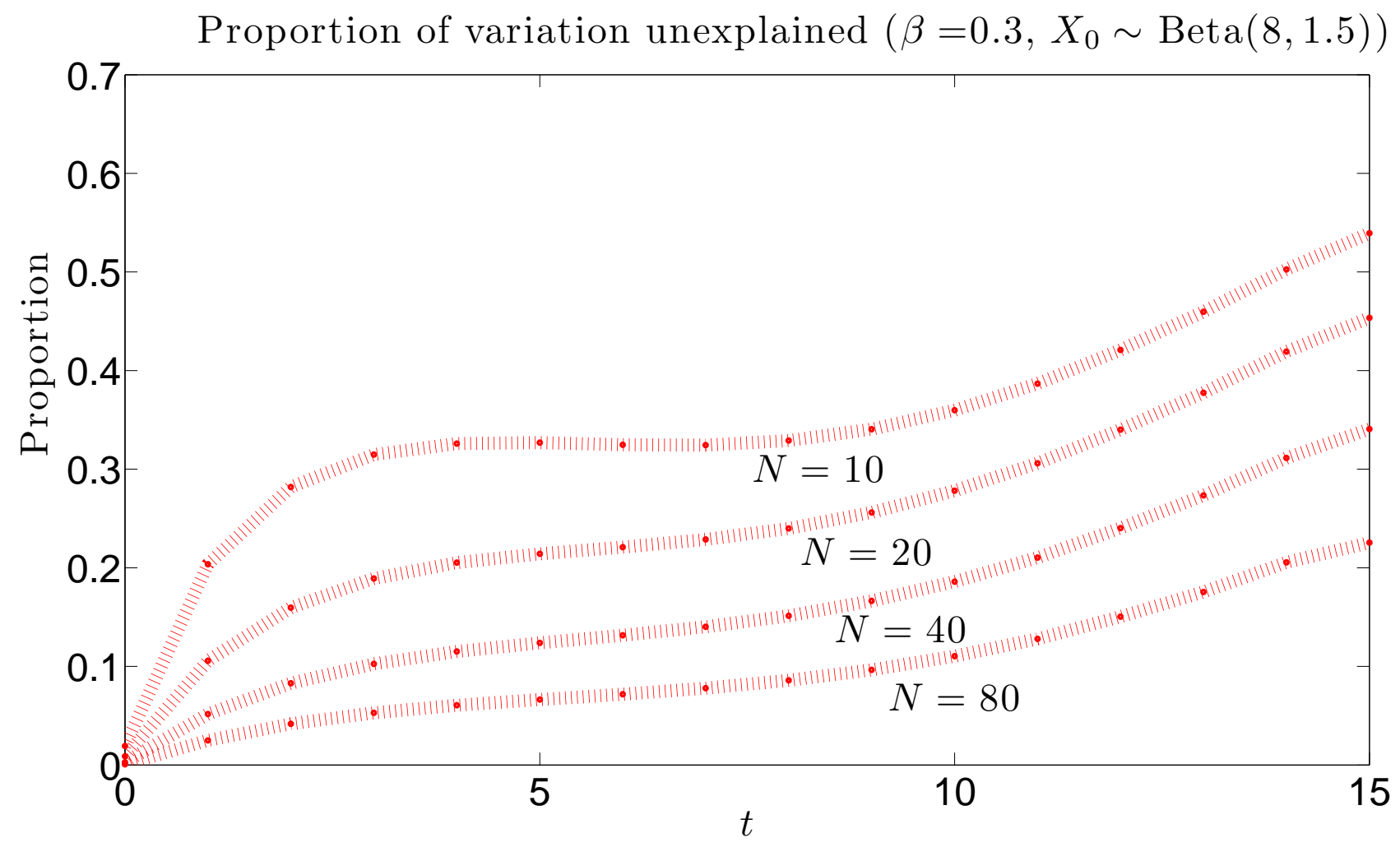


Figure 2 (a)

Simulations and approximations $\left(\beta=0.5, X_{0} \sim \operatorname{Beta}(20,1)\right)$

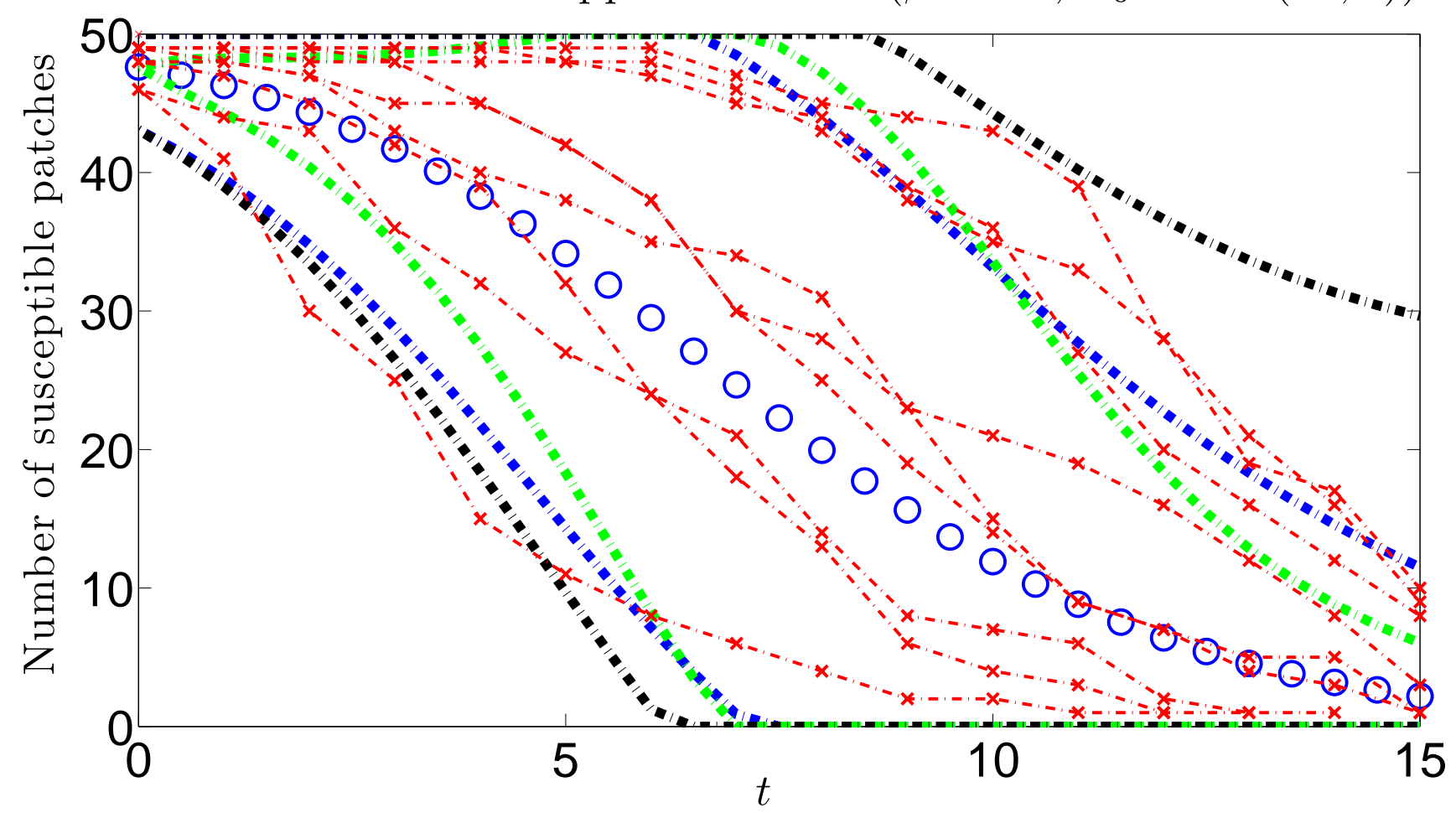

Figure 2 (b)

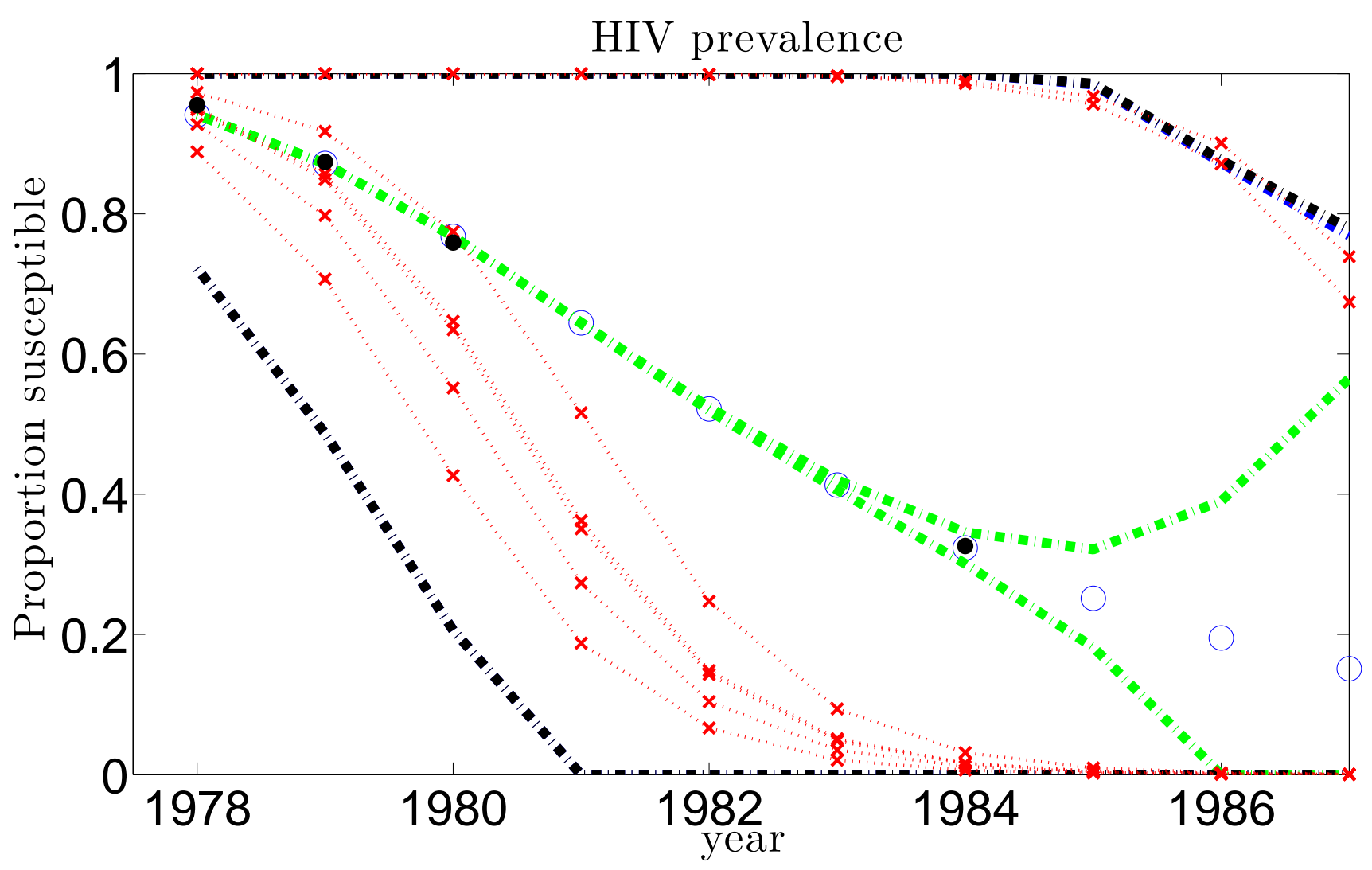


Figure 2 (c)

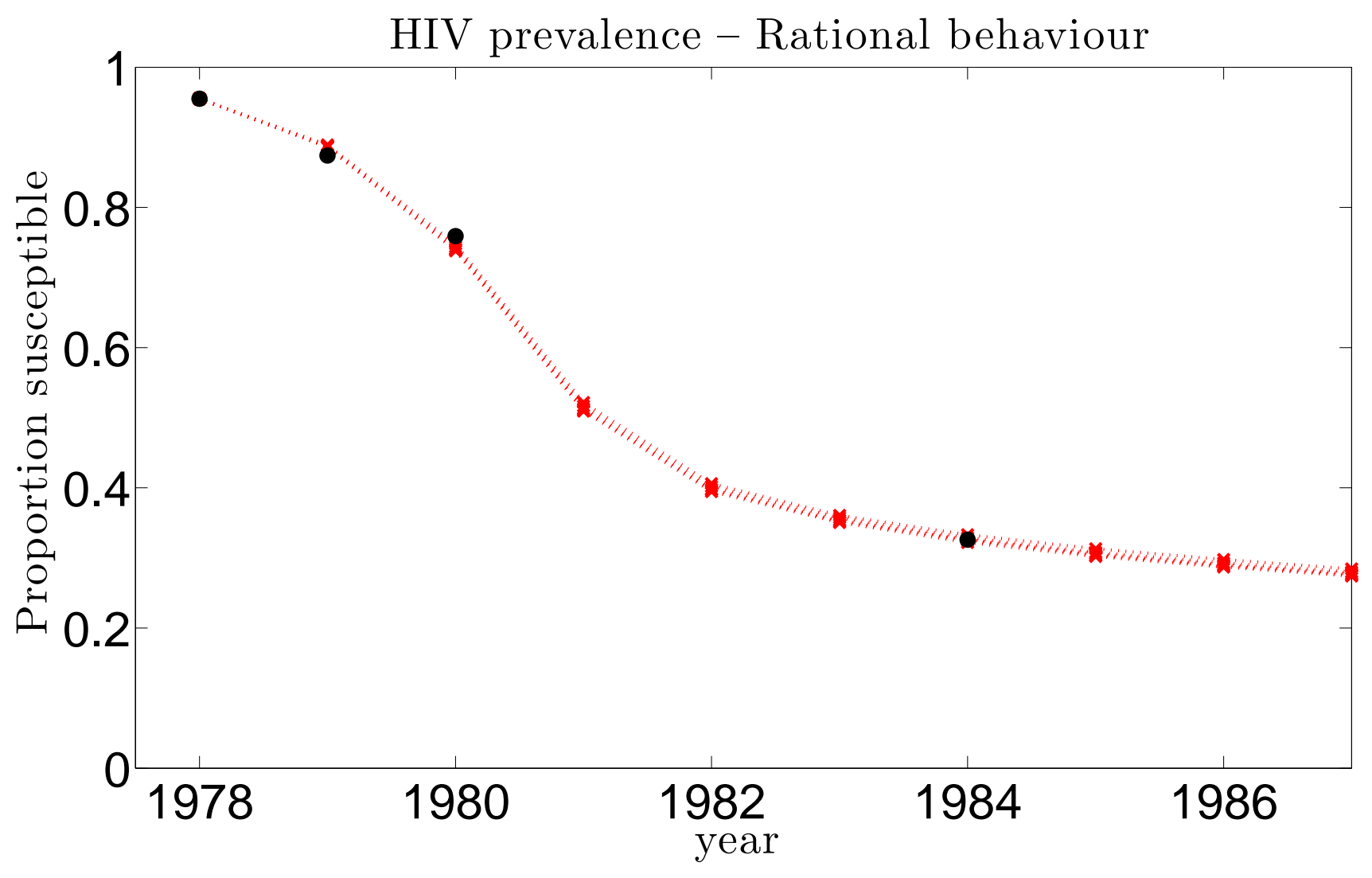

